\title{
An Optimal Algorithm for Tiling the Plane with a Translated Polyomino
}

\author{
Andrew Winslow \\ Université Libre de Bruxelles, 1050 Bruxelles, Belgium, \\ andrew.winslow@ulb.ac. be
}

\begin{abstract}
We give a $O(n)$-time algorithm for determining whether translations of a polyomino with $n$ edges can tile the plane. The algorithm is also a $O(n)$-time algorithm for enumerating all regular tilings, and we prove that at most $\Theta(n)$ such tilings exist.
\end{abstract}

\section{Introduction}

A plane tiling is a partition of the plane into shapes each congruent to a fixed set of tiles. As the works of M. C. Escher attest, plane tilings are both artistically beautiful and mathematically interesting (see [20] for a survey of both aspects). In the 1960s, Golomb [8] initiated the study of polyomino tiles: polygons whose edges are axis-aligned and unit-length.

Building on work of Berger 2, Golomb [9] proved that no algorithm exists for determining whether a set of polyomino tiles has a plane tiling. Ollinger [17] proved that this remains true even for sets of at most 5 tiles. It is a long-standing conjecture that there exists an algorithm for deciding whether a single tile admits a plane tiling (see [10, 11])

Motivated by applications in parallel computing, Shapiro 21] studied tilings of polyomino tiles on a common integer lattice using translated copies of a polyomino. For the remainder of the paper, only these tilings are considered. Ollinger [17] proved that no algorithm exists for determining whether sets of at most 11 tiles admit a tiling, while Wijshoff and van Leeuwen [22] obtained a polynomial-time-testable criterion for a single tile to admit a tiling. Beauquier and Nivat [1, 7] improved on the result of Wijshoff and van Leeuwen by giving a simpler criterion called the Beauquier-Nivat criterion.

Informally, a tile satisfies the Beauquier-Nivat criterion if it can be surrounded by copies of itself (see Figure 1). Such a surrounding must correspond to a regular tiling (also called isohedral) in which all tiles share an identical neighborhood. Using a naive algorithm, the Beauquier-Nivat criterion can be applied to a polyomino with $n$ vertices in $O\left(n^{4}\right)$ time.

The $O\left(n^{4}\right)$ algorithm of 1 is implicit; the main achievement of $[1$ is a concise characterization of exact tiles, akin to Conway's criterion (see [19]). Gambini and Vuillon [6] gave an improved $O\left(n^{2}\right)$-time algorithm utilizing structural and algorithmic results on words describing boundaries of polyominoes. Around the same time, Brlek, Provençal, and Fédou [3, 4] also used a word-based approach 


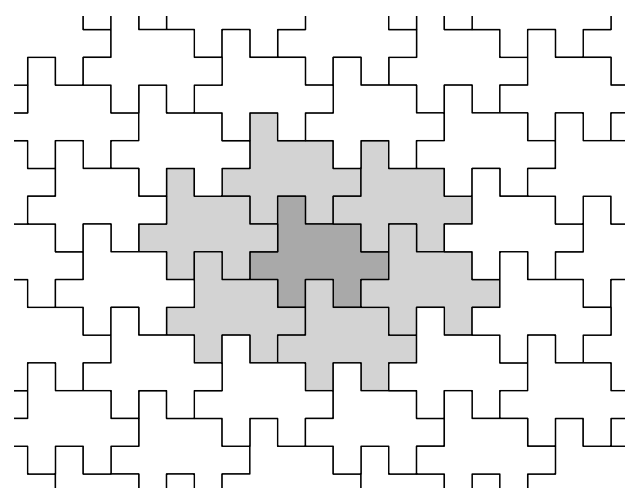

Fig. 1. A polyomino tile (dark gray), a surrounding of the tile (gray), and the induced regular tiling (white).

to achieve $O(n)$-time algorithms for two special cases: (1) the boundary contains no consecutive repeated sections larger than $O(\sqrt{n})$, and (2) testing a restricted version of the Beauquier-Nivat criterion (surroundable by just four copies). Provençal [18 further improved on the algorithm of Gambini and Vuillon for the general case, obtaining $O\left(n \log ^{3}(n)\right)$ running time. In a recent survey of the combinatorics of Escher's tilings, Blondin Massé, Brlek, and Labbé 16. conjecture that a $O(n)$-time algorithm exists. In this work, we confirm their conjecture by giving such an algorithm (Theorem 2).

The algorithm doubles as an algorithm for enumerating all surroundings (regular tilings) of the polyomino. As part of the proof of the algorithm's running time, we prove a claim of Provençal 18 that the number of surroundings of a tile with itself is $O(n)$ (Corollary 1). This complements the tight bounds on a special class of surroundings by Blondin Massé et al. [14, 15, and proves that our $O(n+k)$-time algorithm for enumerating all $k$ surroundings (Lemma 10 is also a $O(n)$-time algorithm.

\section{Definitions}

Here we give precise formulations of terms used throughout the paper. The definitions are similar to those of Beauquier and Nivat [1] and Brlek et al. [4.

\subsection{Words}

A letter is a symbol $x \in \Sigma=\{\mathbf{u}, \mathbf{d}, \mathbf{l}, \mathbf{r}\}$. The complement of a letter $x$, written $\bar{x}$, is defined by the following bijection on $\Sigma: \overline{\mathbf{u}}=\mathbf{d}, \overline{\mathbf{r}}=\mathbf{l}, \overline{\mathbf{d}}=\mathbf{u}$, and $\mathbf{l}=\mathbf{r}$.

A word is a sequence of letters and the length of a word $W$, denoted $|W|$, is the number of letters in $W$. For an integer $i \in\{1,2, \ldots,|W|\}, W[i]$ refers to the $i$ th letter of $W$ and $W[-i]$ refers to the $i$ th from the last letter of $W$. The 
notation $l^{k}$ or $W^{k}$ denotes the word consisting of $k$ repeats of a letter $l$ or word $W$, respectively.

There are several functions mapping a word $W$ to another word of the same length. The complement of $W$, written $\bar{W}$, is the word obtained by replacing each letter of $W$ with its complement. The reverse of $W$, written $\widetilde{W}$, are the letters of $W$ in reverse order. The backtrack of $W$, written $\widehat{W}$, is defined as $\widehat{W}=\overline{\widetilde{W}}$. Note that for any two words $X$ and $Y, \widehat{A B}=\widehat{B} \widehat{A}$.

\subsection{Factors}

A factor of $W$ is an occurrence of a word in $W$, written $X \preceq W$. For integers $1 \leq i, j \leq|W|$ with $i \leq j, W[i . . j]$ denotes the factor of $W$ from $W[i]$ to $W[j]$, inclusive. A factor $X$ starts or ends at $W[i]$ if $W[i]$ is the first or last letter of $X$, respectively.

Two factors $X, Y \preceq W$ may refer the same letters of $W$ or merely have the same letters in common. In the former case, $X$ and $Y$ are equal, written $X=Y$, while in the latter, $X$ and $Y$ are congruent, written $X \equiv Y$. For instance, if $W=$ uuulruuu then $W[1 . .3] \equiv W[6 . .8]$. A factorization of $W$ is a partition of $W$ into consecutive factors $F_{1}$ through $F_{k}$, written $W=F_{1} F_{2} \ldots F_{k}$.

\subsection{Special words and factors}

A word $X$ is a prefix or suffix of a word $W$ provided $W=X U$ or $W=U X$, respectively. A word $X$ is a period of $W$ provided $|X| \leq|W|$ and $W$ is a prefix of $X^{k}$ for some $k \geq 1$ (introduced in [13]). Alternatively, $X$ is a prefix of $W$ and $W[i]=W[i+|X|]$ for all $1 \leq i \leq|W|-|X|$.

A factor $X \preceq W$ is a prefix if $X$ starts at $W[1]$, written $X \preceq$ pre $W$. Similarly, $X \preceq W$ is a suffix if $X$ ends at $W[-1]$, written $X \preceq$ suff $W$. A factor $X \preceq W$ that is either a prefix or suffix is an affix, written $X \preceq$ aff $W$. A factor $X \preceq W$ that is not an affix is a middle, written $X \preceq$ mid $W$.

The factor $X \preceq W$ such that $W=U X V,|U|=|V|$, and $|X| \in\{1,2\}$ is the center of $W$. A factor $X \preceq W$ is a mirror, written $X \preceq$ mir $W$, provided $W=X U Y V$ with $Y \equiv \widehat{X}$ and $|U|=|V|$. For any $X \preceq_{\text {mir }} W, \widehat{X}$ refers to the factor $Y$ in the definition.

A mirror factor is admissible provided $U[1] \neq \overline{U[-1]}, V[1] \neq \overline{V[-1]}$. Observe that each admissible factor is the maximum-length mirror factor with its center. Thus any two admissible factors have distinct centers.

\subsection{Polyominoes and boundary words}

A cell is a unit square with lower-leftmost vertex $(x, y) \in \mathbb{Z}^{2}$ and remaining vertices $(x+1, y),(x, y+1),(x+1, y+1)$. A polyomino is a simply connected union of cells whose boundary is a simple closed curve.

The boundary of a polyomino consists of cell edges. The boundary word of a polyomino $P$, denoted $\mathcal{B}(P)$, is the circular word of letters corresponding to 
the sequence of directions traveled along cell edges during a clockwise traversal of the polyomino's boundary (see Figure 2).

Boundary words are circular: the last and first letters are defined to be consecutive. Thus for any indices $i, j \in \mathbb{Z} \backslash\{0\}, W[i]$ and $W[i . . j]$ are defined. For the boundary word $W=$ urrdll, $W[10]=W[-9]=\mathbf{d}$ and $W[6 . .2]=\mathbf{l u r}$.
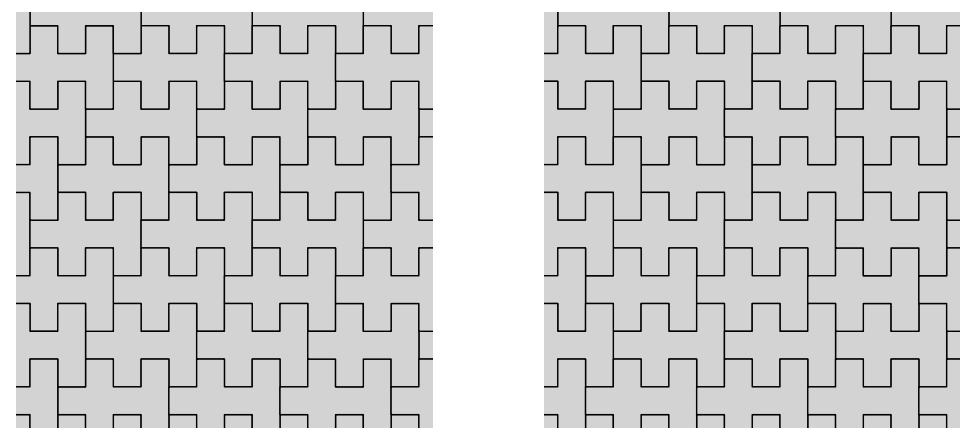

Fig. 2. A regular tiling (left) and non-regular tiling (right) of a polyomino with boundary word ururdrurd ${ }^{3}$ luldlul. The copies in the regular tiling have a common neighborhood factorization $A B C \widehat{A} \widehat{B} \widehat{C}$, with $A=\mathbf{u}, B=\mathbf{r u}, C=$ rdrurd.

\section{$2.5 \quad$ Tilings}

For a polyomino $P$, a tiling of $P$ is an infinite set $\mathscr{T}$ of translations of $P$, called copies, such that every cell in the plane is in exactly one copy. A tiling is regular (e.g. isohedral) provided there exist vectors $\vec{o}, \vec{u}, \vec{v}$ such that the set of lowerleftmost vertices of copies in the tiling is $\vec{o}+\{i \vec{u}+j \vec{v}: i, j \in \mathbb{Z}\}$. Two tilings $\mathcal{T}$ and $\mathcal{T}^{\prime}$ are equal provided there exists a vector $\vec{v}$ such that $\mathcal{T}^{\prime}=\vec{v}+\mathcal{T}$.

Copies of a tiling intersect only along boundaries, and copies with non-empty boundary intersection are neighbors. Lemma 3.5 of [22] implies that the intersection between a pair of neighbors corresponds to a neighbor factor of each neighbor's boundary word and these factors form a neighborhood factorization. Every regular tiling has a neighbor factorization common to all copies in the tiling.

\section{The Beauquier-Nivat Criterion}

Recall that $\widehat{X}$ is the reverse complement of $X$. Thus $\widehat{X}$ is the same path as $X$ but traversed in the opposite direction. So any pair of factors $X$ and $\widehat{X}$ appearing on the boundary of a polyomino are translations of each other with the interior of the boundary on opposite sites. Beauquier and Nivat [1] gave the following criterion for determining whether a polyomino tile admits a tiling: 
Definition 1. A factorization $W=A B C \widehat{A} \widehat{B} \widehat{C}$ of a boundary word $W$ is a $\mathrm{BN}$ factorization.

Lemma 1 (Theorem 3.2 of [1]). A polyomino $P$ has a tiling if and only if $\mathcal{B}(P)$ has a $B N$ factorization.

As seen in Figure 3, a BN factorization corresponds to the neighborhood factorization of a regular tiling. We prove this formally by reusing results from the proof of Lemma 1
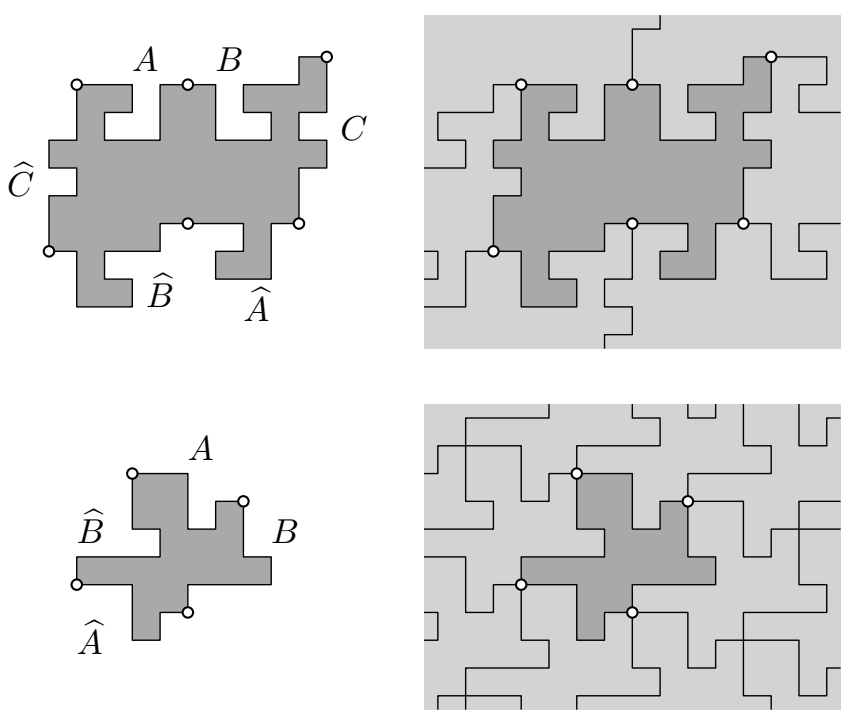

Fig. 3. BN factorizations (left) and the regular tilings induced by these factorizations (right). For one polyomino (bottom), two of the factors are zero length. However, no $\mathrm{BN}$ factorization can have more than two length-0 factors.

Lemma 2 (Corollary 3.2 of [1]). Let $P$ be a polyomino. There exists a factorization $\mathcal{B}(P)=F_{1} \widehat{F_{3}} F_{2} \widehat{F_{1}} F_{3} \widehat{F_{2}}$ if and only if there exists a tiling $\mathscr{T}$ of $P$ with three copies $P_{1}, P_{2}, P_{3}$ such that:

- $P_{1}, P_{2}, P_{3}$ appear clockwise consecutively around a common point $q$.

- $F_{i}$ is the last neighbor factor of $P_{i}$ whose clockwise endpoint is incident to $q$.

Lemma 3. Let $P$ be a polyomino. A factorization of $\mathcal{B}(P)$ is a $B N$ factorization if and only if a regular tiling of $P$ has this neighbor factorization.

Proof. The factorization $\mathcal{B}(P)=F_{1} \widehat{F_{3}} F_{2} \widehat{F_{1}} F_{3} \widehat{F_{2}}$ is a generic BN factorization. So it suffices to prove that there exists a tiling $\mathscr{T}$ of $P$ satisfying the conditions of Lemma 2 if and only if there exists a regular tiling $\mathscr{T}_{\text {reg }}$ with neighbor factorization $\mathcal{B}(P)=F_{1} \widehat{F_{3}} F_{2} \widehat{F_{1}} F_{3} \widehat{F_{2}}$. 
Tiling $\Rightarrow$ neighbor factorization. Let $\mathscr{T}$ be a tiling and $P_{1}, P_{2}, P_{3} \in \mathscr{T}$ be copies as defined in the statement of Lemma 2, Let $\vec{u}$ and $\vec{v}$ be the amount $P_{2}$ and $P_{3}$ are translated relative to $P_{1}$, respectively. Lemma 3.2 of [1] states that the copies obtained by translating $P_{1}$ by $\vec{u}, \vec{v}, \vec{v}-\vec{u},-\vec{u},-\vec{v}$, and $\vec{u}-\vec{v}$ is a surrounding of $P_{1}$ : a set of interior-disjoint copies such that every edge of $C_{1}$ is shared by a copy. Since $P_{3}$ is a copy of $P_{2}$ translated by $\vec{v}-\vec{u}$, the neighbor factor of $P_{1}$ incident to the copy translated by $\vec{v}-\vec{u}$ is $F_{2}$. By similar reasoning, $P_{1}$ has neighbor factors $\widehat{F}_{1}, F_{3}$, and $\widehat{F_{2}}$ incident to the copies translated by $-\vec{u},-\vec{v}$, and $\vec{u}-\vec{v}$, respectively. So $P_{1}$ has neighbor factorization $\mathcal{B}(P)=F_{1} \widehat{F_{3}} F_{2} \widehat{F_{1}} F_{3} \widehat{F_{2}}$. Corollary 3.1 of [1] states that for every surrounding, there exists a regular tiling of $P$ containing the surrounding and thus has the neighbor factorization of $P_{1}$.

Tiling $\Leftarrow$ neighbor factorization. Now suppose there exists a regular tiling $\mathscr{T}_{\text {reg }}$ of $P$ with neighbor factorization $F_{1} \widehat{F_{3}} F_{2} \widehat{F_{1}} F_{3} \widehat{F_{2}}$. Let $P_{1} \in \mathscr{T}_{\text {reg }}$ be a copy and $q$ be the clockwise endpoint of the factor $F_{1}$ of $P_{1}$. Let $P_{2}, P_{3} \in \mathscr{T}_{\text {reg }}$ be copies adjacent to $P_{1}$ and incident to factors $F_{1}$ and $\widehat{F_{3}}$ of $P_{1}$. Let $\vec{u}$ and $\vec{v}$ be the amount $P_{2}$ and $P_{3}$ are translated relative to $P_{1}$, respectively. Then $q$ is the clockwise endpoint of the factor $F_{2}$ of $P_{1}$, translated by $\vec{u}$. Also, $q$ is the clockwise endpoint of the factor $F_{3}$ translated by, translated by $\vec{v}$. So the factors of $P_{2}$ and $P_{3}$ whose clockwise endpoints are $q$ are $F_{2}$ and $F_{3}$, respectively.

\section{A Bound on the Number of Factorizations}

Here we prove that the number of $\mathrm{BN}$ factorizations of the boundary word of an $n$-omino is $O(n)$. This fact is used in Section 4 to improve the bound on the running time the algorithm from $O(n+k)$ to $O(n)$.

Lemma 4. Let $W$ be a boundary word with a factor $X$. Let $P, S \preceq_{\text {mir }} W$ such that $P \preceq_{\text {pre }} X, S \preceq_{\text {suff }} X$, and $P \neq S$. Then $X$ has a period of length $2|X|-$ $(|P|+|S|)$.

Proof. Since $P$ and $S$ are mirror, there exists $X^{\prime} \preceq W$ with $\left|X^{\prime}\right|=|X|, \widehat{P} \preceq_{\text {pre }}$ $X^{\prime}$, and $\widehat{S} \preceq_{\text {suff }} X^{\prime}$. Observe that $X$ has a period of length $r \geq 1$ if and only if $X[i]=X[i+r]$ for all $1 \leq i \leq|X|-r$. Let $1 \leq i \leq|P|+|S|-|X|$. Then $1 \leq|P|+1-i \leq|X|$ and $1 \leq|P|+1+|\widehat{S}|-\left|X^{\prime}\right|-i \leq|\widehat{S}|$. So:

$$
\begin{aligned}
X[i] & =P[i] \\
& =\overline{\widehat{P}}[|P|+1-i] \\
& =\overline{X^{\prime}}[|P|+1-i] \\
& =\overline{\widehat{S}}\left[|P|+1+|\widehat{S}|-\left|X^{\prime}\right|-i\right] \\
& =\overline{\widehat{S}}\left[|\widehat{S}|+1-\left(i+\left|X^{\prime}\right|-|P|\right)\right] \\
& =S\left[i+\left|X^{\prime}\right|-|P|\right] \\
& =X\left[i+\left|X^{\prime}\right|-|P|+(|X|-|S|)\right] \\
& =X[i+2|X|-(|P|+|S|)]
\end{aligned}
$$


Since $P \neq S, 2|X|-(|P|+|S|) \geq 2|X|-(2|X|-1)=1$. So $X$ has a period of length $2|X|-(|P|+|S|)$.

Lemma 5. Let $W$ be a boundary word with $X \preceq W$. Let $P, S \preceq$ mir $W$ such that $P \preceq_{\text {pre }} X, S \preceq$ suff $X$, and $P \neq S$. Any factor $Y \preceq_{\text {mid }} X$ with $|Y|>$ $2|X|-(|P|+|S|)$ is not an admissible factor of $W$.

Proof. By Lemma 4 $X$ has a period of length $r=2|X|-(|P|+|S|)$. Let $Y \preceq_{\text {mid }} X$ and $|Y|>r$.

Let $X^{\prime} \preceq W$ with $\left|X^{\prime}\right|=|X|$ and the center of $X^{\prime}$ exactly $|W| / 2$ letters from the center of $X$. Then $\widehat{P} \preceq_{\text {pre }} X^{\prime}, \widehat{S} \preceq_{\text {suff }} X^{\prime}$, and $\widehat{Y} \preceq_{\text {mid }} X^{\prime}$. Again by Lemma 4. $X^{\prime}$ has a period of length $r$.

Let $U, V \preceq W$ such that $W=Y U \widehat{Y} V$. Since $Y$ is a middle factor of $X$, the letter $U[1]$ is in $X$. Since $X$ has a period of length $r$ and $|Y|>r, U[1]=$ $Y[|Y|+1-r]=\overline{\widehat{Y}[r]}$. Since $\widehat{Y}$ is a middle factor of $X^{\prime}$ and $X^{\prime}$ has a period of length $r, U[-1]=\widehat{Y}[r]$. So $U[1]=\overline{U[-1]}$ and $Y$ is not admissible.

Lemma 6. Let $W$ be a boundary word. There exists a set $\mathscr{F}$ of $O(1)$ factors of $W$ such that every $F \preceq$ adm $W$ with $|F| \geq|W| / 6$ is an affix factor of an element of $\mathscr{F}$.

Proof. A special case on three factors. Let $P_{1}, P_{2}, P_{3} \preceq$ adm $W$ with $\left|P_{1}\right|,\left|P_{2}\right|,\left|P_{3}\right| \geq$ $|W| / 6$ and centers contained in a factor of $W$ with length at most $|W| / 14$. Let $X \preceq W$ be the shortest factor such that $P_{1}, P_{2}, P_{3} \preceq X$, and so $P_{i} \preceq$ pre $X$ and $P_{j} \preceq_{\text {suff }} X$ for some $i, j \in\{1,2,3\}$. We prove that if $i \neq j$, then $P_{1}, P_{2}, P_{3} \preceq_{\text {aff }} X$.

Without loss of generality, suppose $i=1, j=2$ and so $P_{3} \preceq_{\text {mid }} X$. By Lemma 5 since $P_{3} \preceq_{\text {adm }} W,\left|P_{3}\right| \leq 2|X|-\left(\left|P_{1}\right|+\left|P_{2}\right|\right) \leq\left|P_{1}\right|+|W| / 7+\left|P_{2}\right|-$ $\left(\left|P_{1}\right|+\left|P_{2}\right|\right)=|W| / 7<|W| / 6$, a contradiction. So $P_{3} \preceq_{\text {aff }} X$.

All nearby factors. Consider a set $\mathscr{I}=\left\{F_{1}, F_{2}, \ldots, F_{m}\right\}$ of at least three admissible factors of $W$ of length at least $|W| / 6$ such that the centers of the factors are contained in a common factor of $W$ of length $|W| / 14$. We will prove that every element of $\mathscr{I}$ is an affix factor of one of two factors of $W$.

Let $G \preceq W$ be the shortest factor such that $F_{i} \preceq G$ for every $F_{i} \in \mathscr{I}$. It is either the case that there exist distinct $F_{l}, F_{r} \in \mathscr{I}$ with $F_{l} \preceq_{\text {pre }} G, F_{r} \preceq_{\text {suff }} G$, or that $G \in \mathscr{I}$ and every $F_{i} \in \mathscr{I}$ besides $G$ has $F_{i} \preceq_{\text {mid }} G$.

In the first case, $F_{i} \preceq_{\text {aff }} G$ for any $i \neq l, r$ by the previous claim regarding three factors. Also $F_{l}, F_{r} \preceq_{\text {aff }} G$. So every factor in $\mathscr{I}$ is an affix factor of $G$.

In the second case, let $G^{\prime} \preceq G$ be the shortest factor with the same center as $G$ such that every factor in $\mathscr{I}$ excluding $G$ is a factor of $G^{\prime}$. Clearly $G^{\prime} \preceq$ mir $W$ and $G^{\prime} \npreceq$ adm $W$. Without loss of generality, there exists $F_{p} \in \mathscr{I}$ such that $F_{p} \preceq_{\text {pre }} G^{\prime}$. Since $F_{p} \preceq_{\text {adm }} W$ and $G^{\prime} \swarrow_{\text {adm }} W, F_{p} \neq G^{\prime}$.

Applying Lemma 5 with $X=G^{\prime}, P=F_{p}, S=G^{\prime}$, every middle factor of $G^{\prime}$ in $\mathscr{I}$ has length at most $2\left|G^{\prime}\right|-\left(\left|G^{\prime}\right|+\left|F_{p}\right|\right) \leq\left|G^{\prime}\right|-\left|F_{p}\right| \leq|W| / 7<|W| / 6$. So every factor of $G^{\prime}$ in $\mathscr{I}$ is an affix factor of $G^{\prime}$. Thus every factor in $\mathscr{I}$ is either $G$ or an affix factor of $G^{\prime}$.

All factors. Partition $W$ into 15 factors $I_{1}, I_{2}, \ldots, I_{15}$ each of length at most $|W| / 14$. Let $\mathscr{I}_{i}$ be the set of admissible factors with centers containing letters in 
$I_{i}$. Then by the previous claim regarding more than three factors, there exists a set $\mathscr{F}_{i}\left(G\right.$ and possibly $\left.G^{\prime}\right)$ such that every element of $\mathscr{I}_{i}$ is an affix factor of an element of $\mathscr{F}_{i}$ and $\left|\mathscr{F}_{i}\right| \leq 2$. So every $F \preceq$ adm $W$ with $|F| \geq|W| / 6$ is an affix factor of an element of $\mathscr{F}=\bigcup_{i=1}^{15} \mathscr{F}_{i}$ and $|\mathscr{F}| \leq 2 \cdot 15$.

Theorem 1. A boundary word $W$ has $O(|W|) B N$ factorizations.

Proof. Consider the choices for the three factors $A, B, C$ of $\mathrm{BN}$ factorization $W=A B C \widehat{A} \widehat{B} \widehat{C}$. In any factorization, some factor has size at least $|W| / 6$. By Lemma 6 , there exists a $O(1)$-sized set of factors $\mathscr{F}$ such that any factor with length at least $|W| / 6$ is an affix factor of an element of $\mathscr{F}$. Without loss of generality, either $|A| \geq|W| / 6$ and $A$ is a prefix of a factor in $\mathscr{F}$ or $|C| \geq|W| / 6$ and $C$ is a suffix of a factor in $\mathscr{F}$.

Let $H=A B C$ be the factor formed by consecutive factors $A, B, C$ of a BN factorization. Then since $|H|=|W| / 2$ and shares either the first or last letter with a factor in $\mathscr{F}$, there are $O(1)$ total factors $H$. For a fixed $H$, choosing the center of $B$ determines $B$ (since $B$ is admissible) and thus $A$ and $C$. So there are at most $2(|W| / 2)$ factorizations for a fixed factor $H$.

Since Lemma 3 proves that factorizations and tilings are equivalent, the previous theorem implies a linear upper bound on the number of regular tilings of a polyomino:

Corollary 1. An n-omino has $O(n)$ regular tilings.

As pointed out by Provençal [18, it is easy to construct polyominoes with $\Omega(n)$ such tilings. For instance, the polyomino with boundary word $W=\mathbf{u r}^{i} \mathbf{d l}^{i}$ with $i \geq 1$ has $|W| / 2-1$ regular tilings.

\section{An Algorithm for Enumerating Factorizations}

The bulk of this section describes a $O(|W|)$-time algorithm for enumerating the factorizations of a polyomino boundary word $W$. The algorithm combines algorithmic ideas of Brlek, Provençal, and Fédou 4 and a structural result based on a well-known lemma of Galil and Seirferas [5].

Lemma 7 (Corollary 5 of [4]). Every factor of a $B N$ factorization is admissible.

Lemma 8 is a variation of Lemma C4 of Galil and Seirferas [5]. We reproduce their proof with minor modifications.

Lemma 8. Let $A$ and $B$ be two words of the same length. Moreover, let $A=$ $X_{1} X_{2}=Y_{1} Y_{2}=Z_{1} Z_{2}$ and $B=X_{Q} \widehat{X_{2}}=\widehat{Y_{1} Y_{2}}=\widehat{Z_{1}} Z_{Q}$ with $\left|X_{1}\right|<\left|Y_{1}\right|<\left|Z_{1}\right|$. Then $X_{Q}=\widehat{X_{1}}$ and $Z_{Q}=\widehat{Z_{2}}$. 

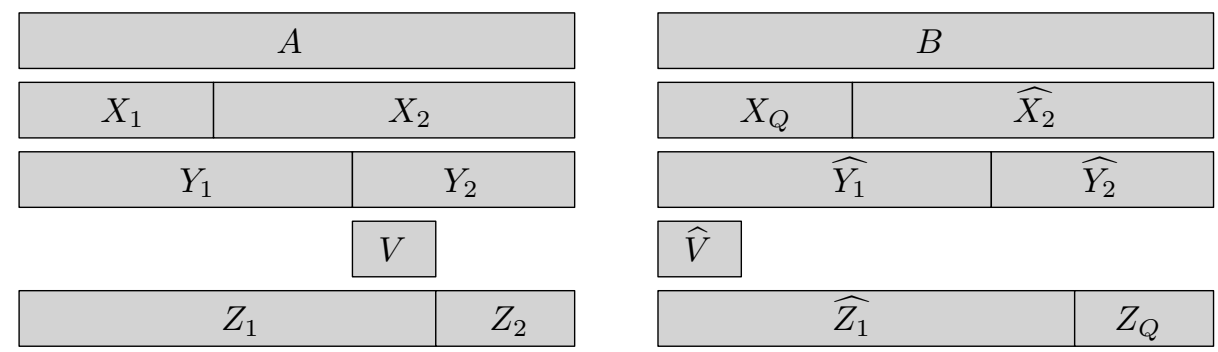

Fig. 4. The words used in the proof of Lemma 8

Proof. Let $V$ be the word such that $Y_{1} V=Z_{1}$ (see Figure 4).

Claim (1): $\widehat{V}$ is a period of $\widehat{Z_{1}}$. Since $Y_{1} V=Z_{1}$, then $\widehat{Z_{1}}=\widehat{Y_{1} V}=\widehat{V} \widehat{Y_{1}}$ is a prefix of $B$. So $\widehat{Y_{1}}$ is a prefix of $\widehat{Z_{1}}=\widehat{V} \widehat{Y_{1}}$ and thus $\widehat{V}$ is a period of $\widehat{Y_{1}}$. So $\widehat{V}$ is a period of $\widehat{V} \widehat{Y_{1}}=\widehat{Z_{1}}$.

Claim (2): $V$ is a prefix of $X_{2}$. Since $V$ is a prefix of $Y_{2}, \widehat{V}$ is a suffix of $\widehat{Y_{2}}$. So $\widehat{V}$ is a suffix of $\widehat{X_{2}}$ and $V$ is a prefix of $X_{2}$.

Claim (3): $X_{1} V$ is a prefix of $Z_{1}$. Since $V$ is a prefix of $X_{2}, X_{1} V$ is a prefix of $Y_{1} V$. Since $\left|X_{1} V\right|<\left|Y_{1} V\right|=\left|Z_{1}\right|, X_{1} V$ is also a prefix of $Z_{1}$.

Claim (4): $\widehat{V}$ is a period of $\widehat{X_{1}}$. By claim (1), $\widehat{V}$ is a period of $\widehat{Z_{1}}$, so $Z_{1}$ has a period of length $|\widehat{V}|=|V|$. By claim (3), $X_{1} V$ is a prefix of $Z_{1}$ and so also has a period of length $|V|$. Then $\widehat{X_{1} V}=\widehat{V} \widehat{X_{1}}$ has a period of length $|V|$, namely $\widehat{V}$. So $\widehat{V}$ is also a period of $\widehat{X_{1}}$.

Finally, combining claims (1) and (4), since $\widehat{V}$ is a period of both $X_{Q}$ and $\widehat{X_{1}}, X_{Q}=\widehat{X_{1}}$. By symmetry, the same proof also implies $Z_{Q}=\widehat{Z_{2}}$.

Lemma 9 (Theorem 9.1.1 of [12]). Two non-circular words $X, Y$ can be preprocessed in $O(|X|+|Y|)$ time to support the following queries in $O(1)$-time: what is the longest common factor of $X$ and $Y$ starting at $X[i]$ and $Y[j]$ ?

Lemma 10. Let $W$ be a polyomino boundary word. Then the BN factorizations of $W$ can be enumerated in $O(|W|)$ time.

Proof. Lemma 7 states that BN factorizations consist entirely of admissible factors. The algorithm first computes all admissible factors, then searches for factorizations consisting of them.

Computing admissible factors. Lemma 7 implies that there are at most $2|W|$ admissible factors, since admissible factor has a distinct center. For each center $W[i . . i]$ or $W[i . i+1]$, the admissible factor with this center is $L R$, where $R$ is the longest common factor of $W$ starting at $W[i+1]$ and $\widehat{W}$ starting at $\widehat{W}[|W| / 2-(i+1)]$. Similarly, $L$ is the longest common factor of $\widetilde{W}$ starting at $\widetilde{W}[|W| / 2-i]$ and $\bar{W}$ starting at $\bar{W}[i]$. Preprocess $W W, \widehat{W} \widehat{W}, \widetilde{W} \widetilde{W}$, and $\bar{W} \bar{W}$ using Lemma 9 so that each longest common factor can be computed in $O(1)$ time. If $|L| \neq|R|$, then $X$ is not admissible and is discarded. Since $O(1)$ time is spent for each of $2|W|$ admissible factors, this step takes $O(|W|)$ total time. 
Enumerating factorizations. Let $W=A Y \widehat{A} Z$ with $A$ an admissible factor and $|Y|=|Z|$. Let $B_{1}, B_{2}, \ldots, B_{l}$ be the admissible prefix factors of $Y$, with $\left|B_{1}\right|<\left|B_{2}\right|<\cdots<\left|B_{l}\right|$. Similarly, let $C_{1}, \ldots, C_{m}$ be the suffix factors with $\left|C_{1}\right|<\cdots<\left|C_{m}\right|$. Lemma 8 implies that for fixed $A$, there exist intervals $[b, l],[c, m]$ such that the $\mathrm{BN}$ factorizations $A B_{i} C_{j} \widehat{A B_{i}} \widehat{C_{j}}$ are exactly those with $i \in[b, l]$ or $j \in[c, m]$.

First, construct a length-sorted list of the admissible factors starting at each $W[k]$ in $O(|W|)$ time using counting sort. Do the same for all factors ending at each $W[k]$.

Next, use a two-finger scan to find, for each factor $A$ that ends at $W[k]$, the longest factor $B_{l}$ starting at $W[k+1]$ such that $|A|+\left|B_{l}\right| \leq|W| / 2$. Then check whether $C_{j}$, the factor following $B_{l}$ such that $\left|A B_{l} C_{j}\right|=|W| / 2$, is admissible and report the factorization $A B_{l} C_{j} \widehat{A B_{l}} \widehat{C_{j}}$ if so. Checking whether $C_{j}$ is admissible takes $O(1)$ time using an array mapping each center to the unique admissible factor with this center.

Additional $\mathrm{BN}$ factorizations containing $A$ are enumerated by checking factors $B_{i}$ with $i=l-1, l-2, \ldots$ for an admissible following factor $C_{j}$. Either $C_{j}$ is admissible and the factorization is reported, or $i=b-1$ and the iteration stops.

Finally, use a similar two-finger scan to find, for each factor $A$ that starts at $W[k]$, the longest factor $C_{m}$ that ends at $W[k+|W| / 2-1]$ such that $|A|+$ $\left|C_{m}\right| \leq|W| / 2$, check whether $B_{i}$ preceeding $C_{m}$ such that $\left|A B_{i} C_{m}\right|=|W| / 2$ is admissible, and report the possible BN factorization. Then check and report similar factorizations with $C_{j}$ for $j=m-1, m-2, \ldots$ until $j=c-1$.

In total, the two-finger scans take $O(|W|)$ time plus $O(1)$ time to report each factorization. Reporting duplicate factorizations can be avoided by only reporting a factorization if $A[1]$ appears before $B[1], C[1], \widehat{A}[1], \widehat{B}[1]$, and $\widehat{C}[1]$ in $W$. Then by Theorem 1 , reporting factorizations also takes $O(|W|)$ time.

Combining this algorithm with Lemmas 1 and 3 yields the desired algorithmic result:

Theorem 2. Let $P$ be a polyomino with n edges. In $O(n)$ time, it can be determined if $P$ admits a tiling and the regular tilings of $P$ can be enumerated.

\section{Acknowledgments}

The author thanks Stefan Langerman for fruitful discussions and comments that greatly improved the paper, and anonymous reviewers for pointing out an error in an earlier version of the paper.

\section{References}

[1] D. Beauquier and M. Nivat. On translating one polyomino to tile the plane. Discrete $\&$ Computational Geometry, 6:575-592, 1991. 
[2] R. Berger. The undecidability of the domino problem. Memoirs of the American Mathematical Society, 66, 1966.

[3] S. Brlek and X. Provençal. An optimal algorithm for detecting pseudo-squares. In A. Kuba, L. G. Nyúl, and K. Palágyi, editors, DGCI 2006, volume 4245 of LNCS, pages 403-412. Springer-Verlag, 2006.

[4] S. Brlek, J.-M. X. Provençal, and Fédou. On the tiling by translation problem. Discrete Applied Mathematics, 157:464-475, 2009.

[5] Z. Galil and J. Seiferas. A linear-time on-line recognition algorithm for "Palstar". Journal of the ACM, 25(1):102-111, 1978.

[6] L. Gambini and L. Vuillon. An algorithm for deciding if a polyomino tiles the plane by translations. RAIRO - Theoretical Informatics and Applications, 41(2):147$155,2007$.

[7] D. Girault-Beauquier and M. Nivat. Tiling the plane with one tile. In 6th Annual Symposium on Computational Geometry, pages 128-138, 1990.

[8] S. W. Golomb. Polyominoes. Scribner's, 1965.

[9] S. W. Golomb. Tiling with sets of polyominoes. Journal of Combinatorial Theory, 9(1):60-71, 1970.

[10] C. Goodman-Strauss. Open questions in tilings. preprint, 2000.

[11] C. Goodman-Strauss. Can't decide? Undecide! Notices of the American Mathematical Society, 57:343-356, 2010.

[12] D. Gusfield. Algorithms on Strings, Trees, and Sequences: Computer Science and Computational Biology. Cambridge University Press, 1997.

[13] D. E. Knuth, J. H. Morris, and V. R. Pratt. Fast pattern matching in strings. SIAM Journal on Computing, 6(2):323-350, 1977.

[14] A. B. Massé, S. Brlek, A. Garon, and S. Labbé. Christoffel and fibonacci tiles. In S. Brlek, C. Reutenauer, and X. Provençal, editors, DGCI 2009, volume 5810 of LNCS, pages 67-78. Springer, 2009.

[15] A. B. Massé, S. Brlek, A. Garon, and S. Labbé. Every polyomino yields at most two square tilings. In 7th International Conference on Lattice Paths and Applications (Lattice Paths 2010), pages 57-61, 2010.

[16] A. B. Masseé, S. Brlek, and S. Labbé. Combinatorial aspects of Escher tilings. In 22nd International Conference on Formal Power Series and Algebraic Combinatorics (FPSAC 2010), pages 533-544, 2010.

[17] N. Ollinger. Tiling the plane with a fixed number of polyominoes. In A. H. Dediu, A. M. Ionescu, and C. Martín-Vide, editors, LATA 2009, volume 5457 of LNCS, pages 638-647. Springer, 2009.

[18] X. Provençal. Combinatoire des mots, géométrie discrète et pavages. $\mathrm{PhD}$ thesis, Université du Québec à Montréal, 2008.

[19] D. Schattschneider. Will it tile? try the Conway criterion! Mathematics Monthly, 53(4):224-233, 1980.

[20] D. Schattschneider. Visions of Symmetry: Notebooks, Periodic Drawings, and Related Work of M. C. Escher. W. H. Freeman and Company, 1990.

[21] H. D. Shapiro. Theoretical limitations on the efficient use of parallel memories. IEEE Transactions on Computers, 27(5):421-428, 1978.

[22] H. A. G. Wijshoff and J. van Leeuwen. Arbitrary versus periodic storage schemes and tessellations of the plane using one type of polyomino. Information and Control, 62:1-25, 1984. 